Cite this: J. Mater. Chem. B, 2014, 2, 1565

Received 17th October 2013 Accepted 2nd January 2014

DOI: $10.1039 / c 3 t b 21454 k$

www.rsc.org/MaterialsB

\section{Poly(ethylene glycol)-block-poly(4-vinyl pyridine) as a versatile block copolymer to prepare nanoaggregates of superparamagnetic iron oxide nanoparticles $\uparrow$}

\author{
Nuno Rocha, ${ }^{\text {a }}$ Joana Mendes, ${ }^{a}$ Luisa Durães, ${ }^{a}$ Hajar Maleki, ${ }^{a}$ António Portugal, ${ }^{a}$ \\ Carlos F. G. C. Geraldes, ${ }^{\text {bcd }}$ Arménio Serra ${ }^{\text {ae }}$ and Jorge Coelho*a
}

This work reports an efficient method for the preparation of aqueous dispersions of superparamagnetic iron oxide nanoparticles (SPIONs), involving the use of an amphiphilic block copolymer, poly(ethylene glycol)block-poly(4-vinyl pyridine) (mPEG-b-P4VP). The iron oxide nanoparticles are easily and efficiently dispersed due to the strong direct interaction of the hydrophobic P4VP segments, through complexation with pyridine units of the copolymer. Well-defined block copolymers, having different compositions and molecular weights, were prepared by atom transfer radical polymerization (ATRP). The aqueous selfassembly behavior of each system has been compared based on the method of preparation. The results revealed that the addition of ionic species has a significant effect on the size and type of formed nanostructures, the magnitude of which is dependent on the block copolymers' molecular design. When similar self-assembly strategies were used in the presence of SPIONs, the same type of nanostructures was formed. The hybrid SPION nanoaggregates were investigated using NMR relaxometric techniques, whereby high $r_{2} / r_{1}$ relaxivity ratios were achieved, making these materials potentially efficient $T_{2^{-}}$ weighted MRI contrast agents

\section{Introduction}

The encapsulation of inorganic species by the self-assembly of amphiphilic block copolymers is a powerful technique for the preparation of inorganic-based nanoparticles having controlled structures and properties. ${ }^{\mathbf{1 , 2}}$ Controlled/living radical polymerization (CLRP) methods ${ }^{3,4}$ are powerful methods for preparing

${ }^{a}$ Department of Chemical Engineering, University of Coimbra, Polo II, Rua Silvio Lima, 3030-790, Coimbra, Portugal. E-mail: nrocha@eq.uc.pt; jcoelho@eq.uc.pt

${ }^{b}$ Department of Life Sciences, Faculty of Sciences and Technology, University of Coimbra, P.O. Box 3046, 3001-401, Coimbra, Portugal

${ }^{c}$ Center of Neurosciences and Cell Biology, Largo Marquês de Pombal, University of Coimbra, Portugal

${ }^{d}$ Coimbra Chemistry Center, Rua Larga, University of Coimbra, 3004-535, Coimbra, Portugal

${ }^{e}$ Chemistry Department, University of Coimbra, 3004-535, Coimbra, Portugal

$\dagger$ Electronic supplementary information (ESI) available: GPC traces that were obtained for the mPEG- $b$-P4VP block copolymers obtained from (a) $\mathrm{mPEG}_{45}-\mathrm{Cl}$ and (b) $\mathrm{mPEG}_{113}-\mathrm{Cl}$ macroinitiators (Fig. S1); ${ }^{1} \mathrm{H}$ NMR spectrum of the mPEG45- $b$-P4VP56-Cl block copolymer (Fig. S2); PPEG $_{113}-b$-P4VP 40 ATRP kinetic plots of (a) conversion and $\ln [\mathrm{M}]_{0} /[\mathrm{M}]$ vs. time and plot of (b) number average molecular weights $\left(M_{\mathrm{n}, \mathrm{GPC}}\right)$ and $M_{\mathrm{w}} / M_{\mathrm{n}} v s$. conversion (\%) in IPA (Fig. S3); particle size distribution in intensity determined by DLS for $\mathrm{MPEG}_{113}-b-\mathrm{P}_{4} \mathrm{VP}_{40}$ under different $\mathrm{pH}$ conditions (Fig. S4). Hydrodynamic diameter $\left(D_{\mathrm{h}}\right)$ of $\mathrm{mPEG}_{113}-b-\mathrm{P}_{4} \mathrm{VP}_{78}$ self-assembled in the presence of hSPIONs measured at different $\mathrm{pH}$ values using the titration method (Fig. S5). See DOI: $10.1039 / \mathrm{c} 3 \mathrm{tb} 21454 \mathrm{k}$ tailor-made telechelic polymers and related amphiphilic block copolymers that have well controlled molecular weights, composition and architecture, under mild reaction conditions..$^{5-9}$ In the presence of inorganic species, the self-assembly of amphiphilic block copolymers can be used to afford hybrid nanoparticles that possess controlled nanostructured morphology. ${ }^{\mathbf{1 0}}$

The coating of magnetic nanoparticles with polymers has often been assumed to be an efficient means of avoiding agglomeration on the basis of electrostatic or static repulsion. ${ }^{11,12}$ This process enhances the particle stability that is of great importance when, for instance, the magnetic nanoparticles are intended to be used in biomedical applications. ${ }^{13-19}$ This aspect is critical for the determination of blood circulation pathways, since the biodistribution of nanoparticles depends on their hydrodynamic sizes and their stability. ${ }^{18}$

Block copolymers have been regarded as an efficient means to prepare size-controlled and aqueous-stable clusters of magnetic nanoparticles, as long as they include, at least, one hydrophilic block for steric stabilization and one anchoring block with affinity for the nanoparticles. ${ }^{20-22}$ The possibility of preparing block copolymers with an efficient control of their molecular structure through CLRP methodologies may be of great significance to control the final structure of the magnetic nanoparticle aggregates. In fact, depending on the block copolymer molecular design and on the self-assembly 
conditions, in the presence of the magnetic nanoparticles, it has been shown that it is possible to obtain nanoaggregates with different morphological properties, such as aggregation number, size and shape, and, therefore, with different magnetic performances. ${ }^{21,23,24}$

For the preparation of stable superparamagnetic iron oxide nanoparticle (SPION) agglomerates by co-micellization in the presence of block copolymers, the selection of the anchoring segment has been mainly based on the chemical nature of the surface of the original SPIONs. Therefore, the nature of the block copolymer that is used to form the SPION nanoaggregates through the self-assembly of block copolymers is determined by the type of interactions that are established between SPIONs and the block copolymer. Amphiphilic block copolymers are usually used when the SPIONs have a hydrophobic coating, being encapsulated into core-shell structures through van der Waals interactions with the hydrophobic polymeric segment. ${ }^{25-27}$ Additionally, double hydrophilic block copolymers can be used to form SPION nanoaggregates, if one of the hydrophilic blocks can establish electrostatic interactions with the SPIONs' surface, such as cationic polymers for negatively charged stabilized SPIONs ${ }^{24,28}$ or anionic polymeric segments for positively charged uncoated iron oxide nanoparticles. ${ }^{\mathbf{2 0} 29}$ Alternatively, the possibility of chemisorbing poly(glycerol (meth)acrylate) onto the SPION's surface through 1,2-diol groups ${ }^{30}$ has been used to form aggregates of block copolymers with SPIONs, but these were limited to self-assembly at the hydrophilic surface of the block copolymers. ${ }^{30,31}$ These SPIONs' agglomerates have been found to be excellent contrast agents for magnetic resonance imaging (MRI) applications. ${ }^{25-28,31}$

Although poly(4-vinyl pyridine) (P4VP) is known to be a strong coordinator for metallic species, ${ }^{32-35}$ it has not yet been used for the stabilization of SPIONs in aqueous medium. We thought that the use of P4VP segments may offer the possibility of direct complexation of pyridyl groups with iron oxide moieties and further interaction through van der Waals forces with, if existing, hydrophobic groups at the SPIONs' surface. When P4VP is combined with a hydrophilic segment in a block copolymer, well-defined core-shell nanoparticles can be formed through a self-assembly mechanism in aqueous medium. ${ }^{36,37}$ Moreover, the strong coordinating nature of the pyridyl moieties in P4VP-based amphiphilic block copolymers has been explored for the preparation of complex structures such as the hydrophilic coating of capillary walls ${ }^{38}$ or $\mathrm{J}$ - and $\mathrm{H}$-aggregates with anionic porphyrins. ${ }^{39}$ In addition, the aqueous selfassembly of P4VP-based amphiphilic block copolymers has been used to prepare water dispersions of hybrid metal-polymer nanoparticles. ${ }^{40}$ The micellization of these amphiphilic P4VP-based block copolymers in the presence of SPIONs, through a self-assembly mechanism, can, thus, be expected to lead to uniformly sized and highly stable nanoaggregates. The possibility of tuning the type of structures that are formed based on the molecular design of the block copolymer and on the selfassembly conditions may be particularly relevant for the preparation of new magnetic-responsive materials, such as for coating applications or for the preparation of MRI contrast agents. $^{41}$
In this paper, we report a novel approach for preparing SPION hybrid core-shell nanoparticles, based on the selfassembly of amphiphilic poly(ethylene glycol)-block-poly(4-vinyl pyridine) (mPEG- $b$-P4VP) and on the transition metal complexation of the pyridyl groups with iron. In addition, the hydrophobic character of the P4VP block, at $\mathrm{pH}$ values above its $\mathrm{p} K_{\mathrm{a}}$, can provide an efficient way to form core-shell nanostructures even with hydrophilic SPIONs or to provide enhanced stabilization for hydrophobic-coated SPIONs, making mPEG- $b$ P4VP a versatile block copolymer to prepare magnetic nanoaggregates for a broad range of SPIONs. This paper compares the aggregation behavior depending on the composition and molecular weight of the block copolymers and on the original SPION surface functionality. In addition, the influence of the self-assembly methodology (titration and solvent exchange method) is investigated. The in vitro performance of the prepared SPIONs' nanoaggregates as potential MRI contrast agents was further assessed and qualitatively related to the structure of their aggregates.

\section{Experimental}

\subsection{Materials}

Each poly(ethylene glycol) methyl ether (mPEG) (mPEG ${ }_{113}: M_{\mathrm{w}}$ $=5000 \mathrm{Da}$, and $\mathrm{mPEG}_{45}: M_{\mathrm{w}}=2500 \mathrm{Da}$; Sigma-Aldrich) was dried by azeotropic distillation from toluene. 2-Chloropropionyl chloride (CPC) (97\%; Sigma-Aldrich) and $\mathrm{CuCl}_{2}$ (+99\% + extra pure, anhydrous; Acros) were used as supplied. $\mathrm{Cu}(0)$ wire (99\%; Acros) was activated with nitric acid, washed with acetone and dried before use. Isopropanol (IPA) (99.97\%; Fisher Chemical), ethanol (96\%; Panreac), diethyl ether (>99.8\%; Sigma-Aldrich), methanol (>99.85\%; Aldrich), chloroform (99.99\%; Fisher Chemical), dimethylformamide (DMF) (+99.8\%; SigmaAldrich), deuterated chloroform $\left(\mathrm{CDCl}_{3}\right)(+1 \%$ tetramethylsilane (TMS); Euriso-top), sodium hydroxide (pellets QP; Panreac) and hydrochloric acid solution ( $\mathrm{HCl})$ (37\%; Aldrich) were used as received. Milli-Q water (Milli-Q®, Millipore) was obtained by reverse osmosis. 4-Vinylpyridine (4VP) (96\%; Fluka), triethylamine (TEA) (96\%; Sigma-Aldrich) and dichloromethane (DCM) (+99.6\%; Fisher Scientific) were dried and distilled under reduced pressure, prior to use. 4-Dimethylaminopyridine (DMAP) (99\%; ACROS) was previously recrystallized. Tris(2dimethylaminoethyl)amine (Me ${ }_{6}$ TREN) was synthesized according to procedures described in the literature. ${ }^{42}$ For Gel Permeation Chromatography (GPC), poly(methyl methacrylate) (PMMA) standards (Polymer Laboratories) (Acros, 99\%, 70 mesh) and high performance liquid chromatography (HPLC) DMF (HPLC grade; Panreac) were used as received. Fe(III) chloride hexahydrate $\left(\mathrm{FeCl}_{3} \cdot 6 \mathrm{H}_{2} \mathrm{O},>99 \%\right), \mathrm{Fe}(\mathrm{II})$ chloride tetrahydrate $\left(\mathrm{FeCl}_{2} \cdot 4 \mathrm{H}_{2} \mathrm{O},>99 \%\right)$, and 1-butanol were purchased from Merck Chemicals Inc.; toluene, acetone, and ethanol were purchased from Ghataran Shimi T. Co. Cetyltrimethylammonium bromide (CTAB) was purchased from Sigma-Aldrich and used as received. Oleic acid-coated hydrophobic SPIONs (OaSPIONs) with a $5 \pm 1 \mathrm{~nm}$ (TEM conform) average particle size and with $10.1 \mathrm{wt} \%$ of oleic-content, as determined by thermogravimetric analysis, were used as 
received (5 mg mL $\mathrm{mL}^{-1}$ in toluene; Aldrich reference no. 700320). The synthesis of poly(ethylene glycol) monomethyl ether chloride (mPEG-Cl) was through an adaptation of a method reported previously. ${ }^{43}$ An illustrative procedure for the preparation of $\mathrm{mPEG}_{113}-\mathrm{Cl}$ is presented in the ESI. $\dagger \mathrm{mPEG}_{113}-b$-P4VP block copolymers were prepared by Atom Transfer Radical Polymerization (ATRP), using the previously reported $\mathrm{Cu}(0)$ and $\left[\mathrm{CuCl}_{2}\right] /$ $\left[\mathrm{Me}_{6} \mathrm{TREN}\right]=1 / 1$ catalytic system,${ }^{44}$ (the procedure is presented in the ESI $\dagger$ ). Hydrophilic $\mathrm{Fe}_{3} \mathrm{O}_{4}$ SPIONs (hSPIONs) were synthesized using a micro-emulsion technique in which two water in oil microemulsions of the same composition (water/ toluene/CTAB-butanol) were prepared, according to our previous work, ${ }^{45}$ (detailed procedure in the ESI $\dagger$ ).

\subsection{Procedures}

2.2.1 Self-assembly of mPEG- $b$-P4VP block copolymers. The self-assembly of the mPEG- $b$-P4VP block copolymers was carried out by titration and solvent exchange methods to afford $1 \mathrm{mg} \mathrm{mL} \mathrm{m}^{-1}$ of aqueous solutions. In the titration method, the block copolymer was previously dissolved in an aqueous solution of $\mathrm{HCl}(0.01 \mathrm{M})$. The solution was then titrated using a very slow dropwise addition of $0.1 \mathrm{M} \mathrm{NaOH}$ aqueous solution. The change in the $\mathrm{pH}$ with respect to the added volume of the $\mathrm{NaOH}$ solution was recorded. The titration was stopped when the $\mathrm{pH}$ stabilized at 11.5.

In the solvent exchange method, ${ }^{21,27}$ the block copolymer was previously dissolved in DMF at a concentration of $10 \mathrm{mg} \mathrm{mL}^{-1}$. $200 \mu \mathrm{L}$ of the block copolymer solution were then added dropwise to $1.8 \mathrm{~mL}$ of Milli-Q water, under vigorous stirring. The sample was then dialyzed against Milli-Q water for 2 days $(\mathrm{MWCO}=1000 \mathrm{Da})$.

2.2.2 Self-assembly of mPEG- $b$-P4VP block copolymers in the presence of SPIONs. The self-assembly of the mPEG- $b$-P4VP block copolymers, in the presence of SPIONs, was carried out using the procedures described for the block copolymers, to afford $1 \mathrm{mg} \mathrm{mL} \mathrm{mL}^{-1}$ of aqueous solutions containing a 10:1 weight ratio of the block copolymer to SPIONs. The titration method was applied only to the hydrophilic SPIONs, because of the non-dispersibility of the hydrophobically coated OaSPIONs. In this method, the block copolymer and the SPIONs were dissolved at an acidic $\mathrm{pH}$ at a weight ratio of block copolymer to SPIONs of $10: 1$.

In the solvent exchange method, for the hydrophilic SPIONs, $200 \mu \mathrm{L}$ of each block copolymer solution in DMF $\left(10 \mathrm{mg} \mathrm{mL}^{-1}\right)$ were added dropwise to $1.8 \mathrm{~mL}$ of a previously prepared dispersion containing $0.11 \mathrm{mg} \mathrm{mL}^{-1}$ of SPIONs in Milli-Q water. For the hydrophobic SPIONs, a mixture of $40 \mu \mathrm{L}$ of SPION solution in toluene $\left(5 \mathrm{mg} \mathrm{mL}^{-1}\right)$ and $200 \mu \mathrm{L}$ of the block copolymer in DMF (10 $\mathrm{mg} \mathrm{mL}^{-1}$ ) was initially prepared. The required dropwise addition was carried out under vigorous stirring in an ultra-sound bath.

\subsection{Characterization}

Gel permeation chromatography (GPC) was carried out using high performance size-exclusion chromatography (HPSEC), with refractive index (RI) (Knauer K-2301) detection. The column set consisted of a PL $10 \mu \mathrm{L}$ guard column $(50 \times 7.5$ $\left.\mathrm{mm}^{2}\right)$, followed by two MIXED-B PL columns $\left(300 \times 7.5 \mathrm{~mm}^{2}, 10\right.$

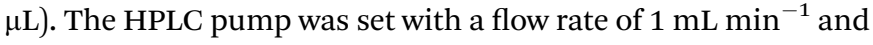
the analyses were carried out at $60{ }^{\circ} \mathrm{C}$ using an Elder $\mathrm{CH}-150$ heater. The eluent was DMF, containing $0.3 \%$ of LiBr. Before injection $(100 \mu \mathrm{L})$, the samples were filtered through a polytetrafluoroethylene (PTFE) membrane with $0.2 \mu \mathrm{m}$ pore size. The system was calibrated against PMMA standards.

$400 \mathrm{MHz}{ }^{1} \mathrm{H}$ NMR spectra of the reaction mixture samples and recovered products were recorded on a Bruker Avance III $400 \mathrm{MHz}$ spectrometer, with a $5 \mathrm{~mm}$ TXI triple resonance detection probe, in $\mathrm{CDCl}_{3}$ using tetramethylsilane (TMS) as an internal standard. NMR measurements were carried out using an acquisition time of $9 \mathrm{~s}$ and a $T_{1}$ of $1 \mathrm{~s}$ (total relaxation delay of $10 \mathrm{~s})$. The conversion of the monomers was determined through the integration of the monomer peak and polymer peak using MestReNova software version: 6.0.2-5475.

Transmission electron microscopy (TEM) was used to observe the size and morphology of the nanoaggregates that were prepared under different self-assembly conditions. Each aqueous dispersion was mounted on a 400 mesh copper grid and examined using a Jeol JEM 1400 transmission electron microscope. Images were digitally recorded using a Gatan SC 1000 ORIUS CCD camera.

Dynamic light scattering (DLS) measurements were performed on a Malvern Instruments Zetasizer Nano-ZS (Malvern Instruments Ltd.). The particle size distribution (in intensity), average hydrodynamic particle size average ( $z$-average) and polydispersity index (PDI) were determined with Zetasizer 6.20 software. Measurements were made at $25{ }^{\circ} \mathrm{C}$ and at a backward scattering angle of $173^{\circ}$. All of the samples were filtered before measurements through a membrane of poly(propylene) (PP) (0.45 $\mu \mathrm{m}$ pore size), to remove any existing dust. At least, 4 measurements were taken for each sample.

Atomic Absorption Spectroscopy (AAS) was used to evaluate the residual copper catalyst content in the block copolymers and the content of iron in the prepared hybrid samples. AAS was performed on a Perkin Elmer (Model 3300). For the copper content determination, the block copolymers were dissolved in a $0.1 \mathrm{M} \mathrm{HCl}$ solution at a known concentration between 0.5 and $1 \mathrm{mg} \mathrm{mL}{ }^{-1}$. The iron content in each self-assembled block copolymer and SPION samples was measured from dilutions of the final dispersion in a $0.1 \mathrm{M} \mathrm{HCl}$ solution. At least, five measurements were taken for each sample.

For the relaxivity measurements of the different SPIONs and block copolymer formulations, the water proton longitudinal $\left(T_{1}\right)$ and transverse $\left(T_{2}\right)$ relaxation times were measured using a Bruker Minispec mq20, operating at a magnetic field of $0.47 \mathrm{~T}$, corresponding to a Larmor frequency of $20 \mathrm{MHz}$, and at a temperature of $25{ }^{\circ} \mathrm{C} . T_{1}$ and $T_{2}$ relaxation times were obtained using the inversion-recovery (IR) and Carr-Purcell-MeiboomGill (CPMG) pulse sequences, respectively. The accuracy of the relaxation time determinations was better than $\pm 1 \%$. For $T_{1}$ determinations, for each measurement a relaxation delay longer than five times $T_{1}$ was used and the interpulse delay was varied between the shortest and longest values, in order to cover the full range of longitudinal magnetization recovery. For $T_{2}$ 
determinations, the same care was taken to choose relaxation delays longer than five times $T_{2}$ and an array of echo times was selected to allow full sampling of the transverse magnetization decay. The paramagnetic contribution to the water proton inverse relaxation times, $R_{i, \mathrm{p}}$, varied linearly with the analytical Fe concentration $[\mathrm{Fe}]$, according to eqn (1). ${ }^{12}$

$$
R_{i, \mathrm{p}}=R_{i, \mathrm{obs}}-R_{i}^{0}=r_{i}[\mathrm{Fe}](i=1,2) .
$$

In eqn (1), $R_{i, \mathrm{obs}}$ and $R_{i}^{0}$ are, respectively, the inverse relaxation times determined experimentally and the inverse relaxation times of aqueous solutions of the block copolymer alone at a concentration corresponding to that existing in the hybrid sample, while the relaxivities $r_{i}(i=1,2)$ are defined as the water proton relaxation rate enhancements per $\mathrm{mM}$ Fe concentration. Different iron concentrations were obtained by the sequential dilution of the original sample. This dilution was performed 3 times to obtain four concentrations.

\section{Results}

\subsection{Synthesis and characterization of mPEG- $b$-P4VP block copolymers}

Block copolymers of mPEG- $b$-P4VP with different compositions and molecular weights were prepared by the ATRP of 4VP using a $\mathrm{Cu}(0) / \mathrm{CuCl}_{2} / \mathrm{Me}_{6}$ TREN catalytic system, in isopropanol at 50 ${ }^{\circ} \mathrm{C}$ (ref. 44) using mPEG-Cl macroinitiators. Table 1 summarizes the polymerization parameters that were used and the characteristics of the obtained block copolymers. The results indicate that MPEG-Cl macroinitiators were successfully initiated to afford mPEG- $b$-P4VP block copolymers, with narrow molecular weight distributions, for the different compositions and molecular weights (see GPC traces in Fig. S1, ESI $\dagger$ ). The ${ }^{1} \mathrm{H}$ NMR spectrum of the $\mathrm{mPEG}_{45}-b-\mathrm{P}_{4} \mathrm{VP}_{56}$ block copolymer (Fig. S2, $\mathrm{ESI} \dagger)$ shows the mPEG's terminal $\mathrm{C}-\left(\mathrm{CH}_{3}\right)$ protons, at $0.80-1.00$ ppm, the P4VP characteristic protons at 1.20-2.00 ppm $(\mathrm{d}, 3 \mathrm{H})$, and its aromatic ring at 6.00-6.90 ppm (e, 2H) and 7.70-8.90 ppm (f, $2 \mathrm{H})$. The average number molecular weight $\left(M_{\mathrm{n}}\right)$ of the P4VP blocks was determined through the comparison of the NMR peak integrals and from GPC measurements. The obtained narrow molecular weight distributions and the kinetic results of polymerizations ${ }^{4}$ (Fig. S3, ESI $\dagger$ ) suggest the success of the ATRP method that was adopted. One possible drawback that can be associated with the use of ATRP to prepare P4VP-containing structures may be the presence of copper in the final product. However, as can be observed in Table 1, the amount of copper in the block copolymers is residual, with values ranging from 0.55 and $0.94 \%$. As an example, for the copolymer $\mathrm{mPEG}_{45}-b-\mathrm{P} 4 \mathrm{VP}_{32}$, this corresponds to only $2.5 \%$ of the existing pyridyl groups complexed with the copper ions (1:1 complex).

\subsection{Preparation and characterization of MPEG- $b$-P4VP micellar structures}

Considering the pH-responsive character of P4VP, one way of preparing the block copolymer micelles involves aqueous selfassembly as a function of $\mathrm{pH}$ change, as previously described for amphiphilic P4VP-based block copolymers. ${ }^{36,37}$ As represented in Scheme 1, these block copolymers are soluble under acidic conditions, but form core-shell micelles when the $\mathrm{pH}$ is above the $\mathrm{p} K_{\mathrm{a}}$ of $\mathrm{P} 4 \mathrm{VP}(\sim \mathrm{pH} 4.5)^{37}$, at which point the P4VP chains are deprotonated and the P4VP segments become hydrophobic. Another route for particle formation is via a solvent exchange method, as schematically represented in Scheme 1. In this method, the block copolymer is initially dissolved in a good solvent for both blocks (such as DMF) and, due to dispersibility changes caused by dropwise addition into water, the particles are formed and are further subjected to dialysis against distilled water. The self-assembly of mPEG- $b$ $\mathrm{P} 4 \mathrm{VP}$ could be monitored by the titration of $\mathrm{HCl}$-acidic aqueous

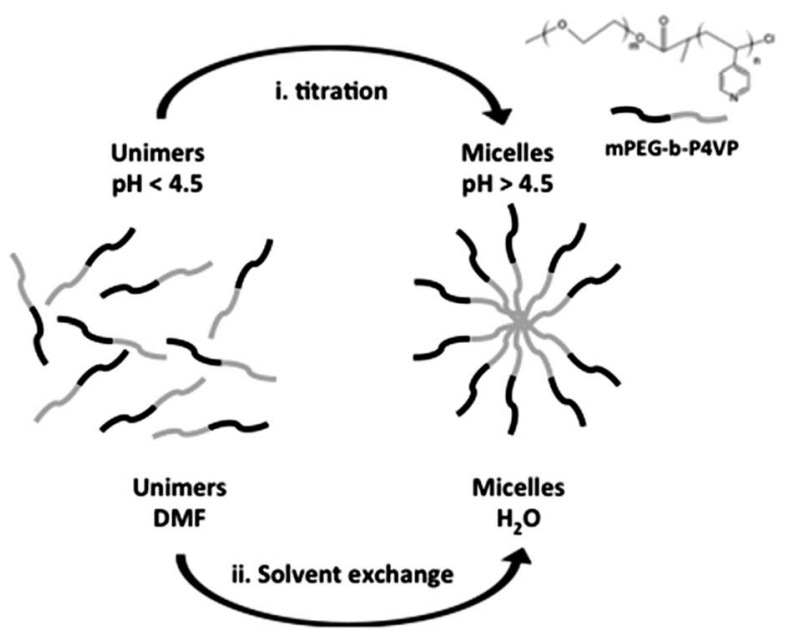

Scheme 1 Representation of mPEG-b-P4VP self-assembly in aqueous media via titration and solvent exchange methods.

Table $1 \bar{M}_{n}$ and $\bar{M}_{n} / \bar{M}_{w}$ values determined by ${ }^{1} \mathrm{H}$ NMR and GPC and residual copper content determined by elemental analysis for the mPEG- $b-$ P4VP block copolymer products

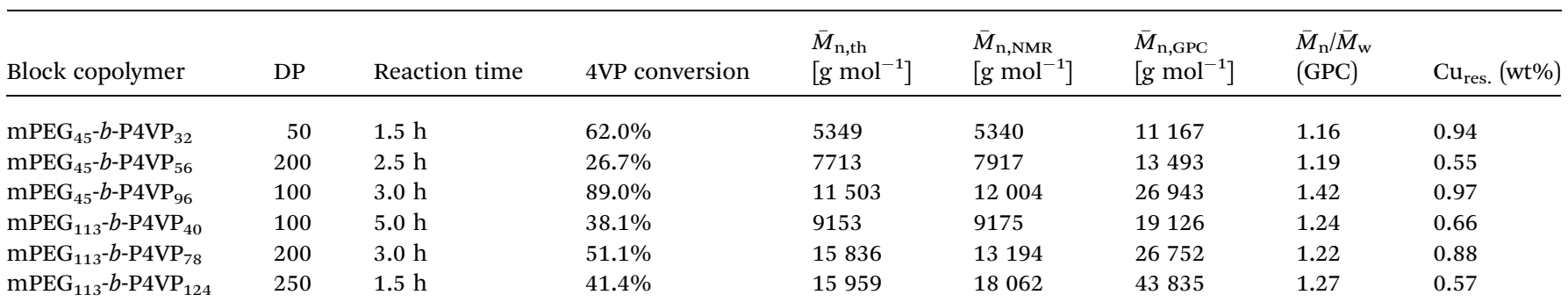


dispersions of the block copolymer, using a $\mathrm{NaOH}$ solution. Change from unimers to the micellized state was indicated by an increase in the solution turbidity when the $\mathrm{p} K_{\mathrm{a}}$ of P4VP was reached, and could be confirmed by the significant increase in their hydrodynamic size, as determined by DLS (Fig. S4, ESI $\dagger$ ). A similar process of particle formation was observed for nanoparticles produced through the use of solvent exchange process. The particle size distributions were determined by DLS and the average hydrodynamic diameters $\left(D_{\mathrm{h}}\right)$ and polydispersity index (PDI) of the nanoparticles obtained are listed in Table 2.

The results presented in Table 2 for the titration method show a tendency towards an increase in the hydrodynamic size with increase in the length of the P4VP block, while maintaining a narrow particle size distribution. The narrow size distributions can be related to the narrow molecular weight distributions of the block copolymers, which are essential for the self-assembly into uniform structures. ${ }^{46}$ When a greater P4VP to $\mathrm{MPEG}$ ratio is used, larger particles with broader distributions were formed. This behavior was less pronounced when a longer mPEG segment was used, with particles of similar sizes being formed. The destabilization of micellar structures by increasing the P4VP block length was also previously observed when the titration method was used for P4VP block copolymers having poly(glycidol) as the steric stabilizer. ${ }^{37}$ For the solvent exchange method, the self-assembly of block copolymers with greater P4VP to $\mathrm{mPEG}$ chain length ratios led to particles that were significantly smaller than those obtained by the titration method.

It should be noted that, in the titration method, the hydrodynamic size of the self-assembled block copolymers is only slightly affected by $\mathrm{pH}$ changes. In fact, specifically for the block copolymer $\mathrm{mPEG}_{45}-b-\mathrm{P}_{4} \mathrm{VP}_{56}$, the slight variation of the measured hydrodynamic size of the particles at different $\mathrm{pH}$ values (Fig. S5†) points out for only a negligible effect of the $\mathrm{pH}$ on the particle size of the self-assembled SPIONs' aggregates using the titration method. Previous studies on the selfassembly of capsules that were formed from P4VP and poly(methacrylic acid $)^{47}$ have shown that, when the ionic strength was increased, the stability of the capsules decreased, leading to an increase in swelling and even to the precipitation of P4VP at higher $\mathrm{pH}$ values.

To further inspect the effect of the ionic strength on the self-assembly of the block copolymers, $\mathrm{mPEG}_{45}-b$-P4VP 56

Table $2 D_{\mathrm{h}}$ and PDI of aqueous block copolymer dispersions, as determined by DLS, prepared by titration and by solvent exchange methods

\begin{tabular}{|c|c|c|c|c|}
\hline \multirow[b]{3}{*}{ Block copolymer } & \multirow{2}{*}{\multicolumn{2}{|c|}{$\begin{array}{l}\text { Titration } \\
\mathrm{pH}=11.5\end{array}$}} & \multirow{2}{*}{\multicolumn{2}{|c|}{$\begin{array}{l}\text { Solvent exchange } \\
\mathrm{pH}=6.0\end{array}$}} \\
\hline & & & & \\
\hline & $D_{\mathrm{h}}(\mathrm{nm})$ & PDI & $D_{\mathrm{h}}(\mathrm{nm})$ & PDI \\
\hline $\mathrm{mPEG}_{45}-b-\mathrm{P} 4 \mathrm{VP}_{32}$ & 24.7 & 0.08 & 17.8 & 0.15 \\
\hline $\mathrm{mPEG}_{45}-b-\mathrm{P} 4 \mathrm{VP}_{56}$ & 93.4 & 0.19 & 29.7 & 0.22 \\
\hline $\mathrm{mPEG}_{45}-b-\mathrm{P} 4 \mathrm{VP}_{96}$ & 613.2 & 0.29 & 17.6 & 0.21 \\
\hline $\mathrm{mPEG}_{113}-b-\mathrm{P}_{4} \mathrm{VP}_{40}$ & 34.0 & 0.21 & 32.0 & 0.18 \\
\hline $\mathrm{mPEG}_{113}-b-\mathrm{P} 4 \mathrm{VP}_{78}$ & 50.9 & 0.10 & 35.7 & 0.09 \\
\hline $\mathrm{mPEG}_{113}-b-\mathrm{P}_{4} \mathrm{VP}_{124}$ & 55.1 & 0.12 & 25.1 & 0.26 \\
\hline
\end{tabular}

nanoparticles were prepared by solvent exchange at different $\mathrm{NaCl}$ ionic strengths. The hydrodynamic size was found to increase from $29.7 \mathrm{~nm}$ (see Table 2) to $110.3 \mathrm{~nm}$ and $93.7 \mathrm{~nm}$ at, respectively, $10 \mathrm{mM}$ and $200 \mathrm{mM}$ of $\mathrm{NaCl}$. These results support the conclusion that the observed increase in the particle size of the samples prepared by the titration method is due to the increase in the ionic strength of the medium.

Fig. 1 presents the TEM micrographs of the self-assembled block copolymers prepared by titration and solvent exchange methods. As shown in Fig. 1a, after titration the block copolymer molecules are seen to be self-assembled into reasonably similar structures, with particle sizes in the $20-40 \mathrm{~nm}$ range. These structures seem to incorporate voids that can be attributed to a swelling effect arising from an increase in the ionic strength that occurs during titration. When the solvent exchange method was applied, only small and compacted aggregates $(20-30 \mathrm{~nm})$ were formed. Interestingly, these structures did not show evidence of large voids that were identified in Fig. 1a. This difference in self-assembled structures suggests that the formation of ionic species during titration interferes significantly with the particle formation process and is responsible for the increased swelling of the nanoparticles.

\subsection{Preparation and characterization of SPION aggregates based on mPEG- $b$-P4VP self-assembly}

Hydrophilic SPIONs (hSPIONs) with an average size of $10 \mathrm{~nm}$ and a cubic morphology were synthesized according to our reported work. ${ }^{45}$ The ability of MPEG- $b$-P4VP to form micellar structures in water can be used to prepare core-shell SPION aggregates that have the SPIONs in their core (Scheme 2). The coordination capacity of P4VP, combined with its hydrophobicity at the self-assembled state, enables the transport of the hSPIONs into the core during micelle formation.

Table 3 presents the hydrodynamic diameter and polydispersity of the aggregates that were obtained by the selfassembly of mPEG- $b$-P4VP in the presence of hSPIONs. The increase in the hydrodynamic size, compared to that corresponding to the block copolymer alone, suggests that the hSPIONs are in the micelle core.

The results that were obtained when the hSPION aggregates were prepared by the titration method followed the same trend as those obtained from the titration of the block copolymers alone (compare with Table 2), since there was a tendency towards a significant increase in the particle size of the self-
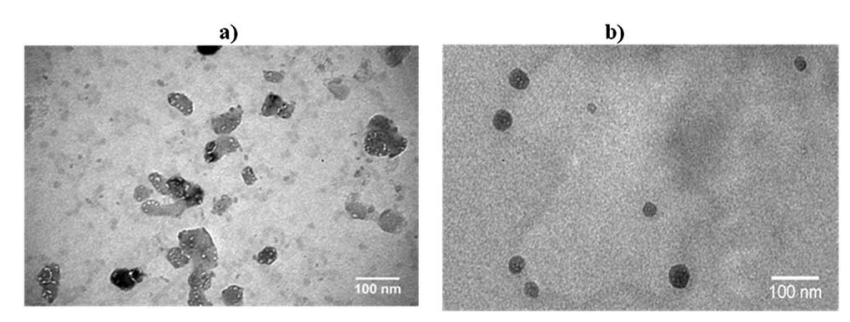

Fig. 1 TEM images of self-assembled block copolymers of (a) $\mathrm{mPEG}_{113}-b-\mathrm{P}_{4} \mathrm{VP}_{40}$ obtained by the titration method and (b) $\mathrm{mPEG}_{45}$ $b-\mathrm{P}_{\mathrm{V}} \mathrm{P}_{96}$ prepared by solvent exchange. 


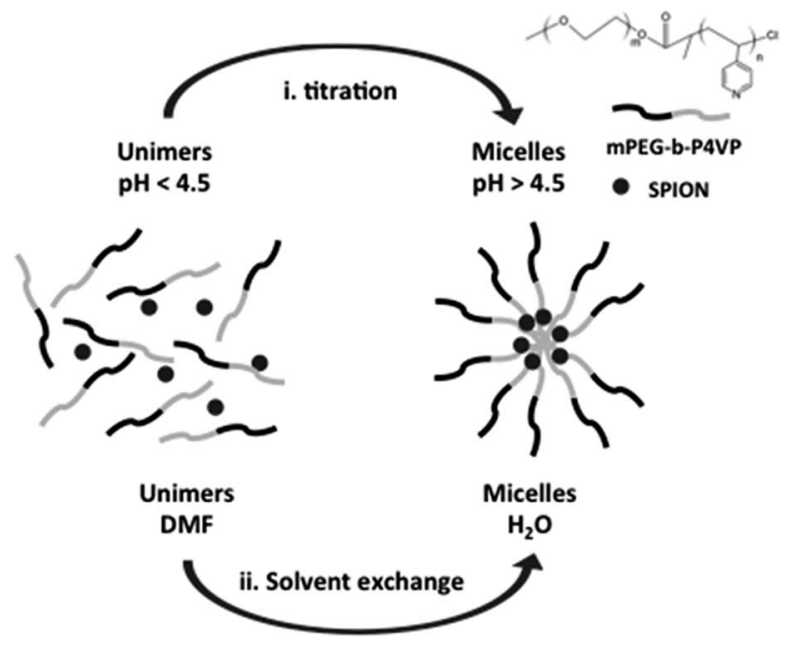

Scheme 2 Schematic representation of the self-assembly of mPEG$b$-P4VP in the presence of $\mathrm{Fe}_{3} \mathrm{O}_{4}$-based SPIONs, via titration and via solvent exchange methods.

Table $3 D_{\mathrm{h}}$ and PDI values for mPEG- $b$-P4VP, self-assembled in the presence of hSPIONs

\begin{tabular}{|c|c|c|c|c|}
\hline \multirow[b]{2}{*}{ Block copolymer } & \multicolumn{2}{|l|}{ Titration } & \multicolumn{2}{|c|}{ Solvent exchange } \\
\hline & $\begin{array}{l}z \text {-Average } \\
D_{\mathrm{h}}(\mathrm{nm})\end{array}$ & PDI & $\begin{array}{l}z \text {-Average } \\
D_{\mathrm{h}}(\mathrm{nm})\end{array}$ & PDI \\
\hline $\mathrm{mPEG}_{45}-b-\mathrm{P} \mathrm{VP}_{32}$ & 66.7 & 0.27 & 66.8 & 0.19 \\
\hline $\mathrm{mPEG}_{45}-b-\mathrm{P}_{4} \mathrm{VP}_{56}$ & 113.1 & 0.20 & 70.2 & 0.25 \\
\hline $\mathrm{mPEG}_{45}-b-\mathrm{P} 4 \mathrm{VP}_{96}$ & Precipitates & & 55.2 & 0.27 \\
\hline $\mathrm{mPEG}_{113}-b-\mathrm{P} 4 \mathrm{VP}_{40}$ & 71.8 & 0.25 & 160.0 & 0.36 \\
\hline $\mathrm{mPEG}_{113}-b-\mathrm{P} 4 \mathrm{VP}_{78}$ & 78.5 & 0.19 & 69.9 & 0.26 \\
\hline $\mathrm{mPEG}_{113}-b-\mathrm{P}_{4} \mathrm{VP}_{124}$ & 86.5 & 0.22 & 68.8 & 0.28 \\
\hline
\end{tabular}

assembled structures as the P4VP to MPEG molar ratio was increased. This observation was particularly relevant for the block copolymer with the lowest MPEG molecular weight. In fact, $\mathrm{mPEG}_{45}-b-\mathrm{P}_{4 \mathrm{VP}} \mathrm{P}_{66}$ could not even form stable aggregates of hSPIONs. This effect may be attributed to the formation of a large core that could not be stabilized by a small hydrophilic segment (recall large structures obtained by titration of $\left.\mathrm{mPEG}_{45}-b-\mathrm{P} \mathrm{VP}_{96}\right)$.

When the solvent exchange method was used there was also a tendency for more compacted structures to be formed when the P4VP to MPEG ratio was increased, while maintaining the low polydispersity. The only exception was obtained for the nanoaggregates prepared with $\mathrm{MPEG}_{113}-b-\mathrm{P}_{4 \mathrm{VP}} \mathrm{P}_{40}$. This increase in the particle size of the hybrid nanoparticles suggests that, at lower P4VP to MPEG chain length ratios, the reduced amount of pyridyl groups that are available leads to an inferior capacity for coordination with the hSPIONs. Therefore, less compacted micelles including non-coordinated aggregates of hSPIONs are expected to be formed.

Fig. 2 provides microscopic evidence for the aggregation of hSPIONs that can be achieved by the two self-assembly methodologies. It is interesting to notice that, similar to what

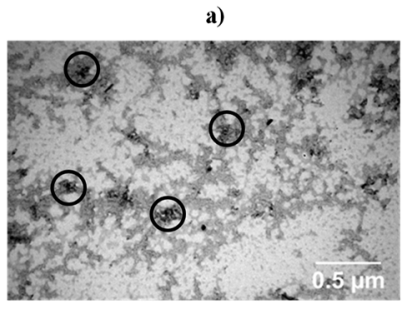

c)

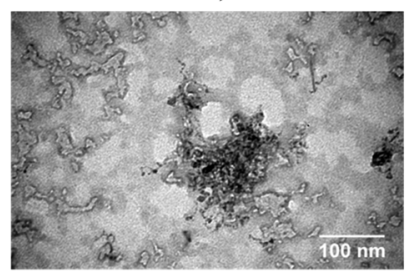

b)

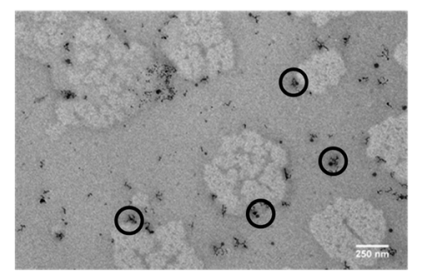

d)

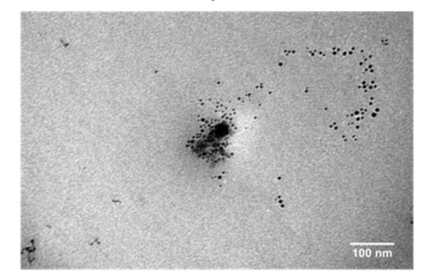

Fig. 2 TEM micrographs of self-assembled block copolymers with hSPIONs prepared with ( $a$ and c) $\mathrm{mPEG}_{113}-b-\mathrm{P}_{4} \mathrm{VP}_{40}$ by titration and (b and d) $\mathrm{mPEG}_{45}-b-\mathrm{P}_{4} \mathrm{VP}_{32}$ by solvent exchange. ( $\mathrm{a}$ and $\mathrm{b}$ ) Micrographs were taken at Mag. $\times 60000$ and highlight the presence of the nanoaggregates (black circles), whereas (c and d) micrographs were taken at Mag. $\times 200000$.

occurred for the self-assembly of block copolymers, the disappearance of the swelled and void-including structures is observed, when the preparation method is changed from the titration to the solvent exchange method. Another significant point is the presence of small particles outside the self-assembled structures. This presence can be attributed to the hydrophilic nature of the hSPIONs, which may favor some particles being outside the self-assembled structures.

To understand the influence of the original coating of the SPIONs on the formation of hybrid nanoaggregates with mPEG$b$-P4VP block copolymers, hydrophobic oleic acid-stabilized SPIONs (OaSPIONs) were evaluated. It should be noted that the hydrophobic nature of OaSPIONs makes it impossible to predisperse the nanoparticles in water and, therefore, only the solvent exchange method could be used. Fig. 3 gives the particle size distributions that were obtained. Similar to what occurred for the self-assembly with hSPIONs, there is an increase in the size of the particle formed by the mPEG- $b$-P4VP self-assembly (compare with Table 2), suggesting an efficient encapsulation of
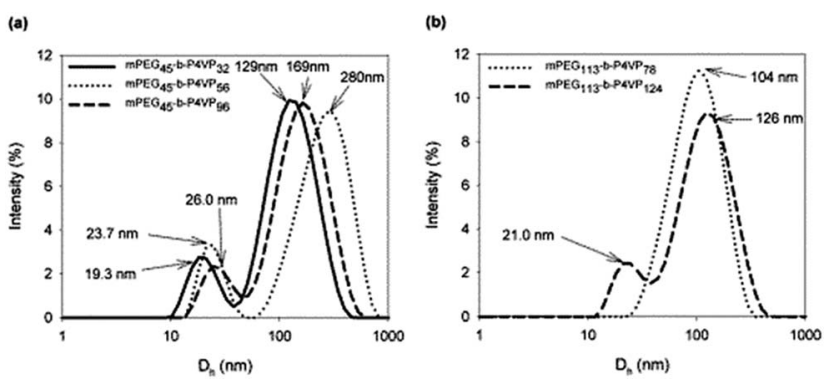

Fig. 3 Particle size distributions, determined by DLS, for MPEG- $b$ P4VP block copolymers that were self-assembled in the presence of OaSPIONs. The block copolymers were based on (a) mPEG $_{45}$ and (b) $\mathrm{mPEG}_{113}$ respectively as the steric stabilizer. 
the SPIONs in the micellar core. The size distribution of $\mathrm{mPEG}_{113}-b-\mathrm{P}_{4 \mathrm{VP}}$ 4 could not be determined because of the presence of large aggregates. In fact, this block copolymer gave larger hybrid nanoaggregate particle sizes when the solvent exchange method was used and such behaviour was attributed to the reduced amount of P4VP. Thus, the precipitating behavior that was observed for this particular copolymer may be explained by a reduced ability to stabilize the core of the aggregates, when the SPIONs have a hydrophobic coating.

Additionally, when the OaSPIONs were used, bimodal size distributions were found in most of the samples. Since OaSPIONs cannot be dispersed in water, because of their hydrophobic character, the smaller sized population $(20-30 \mathrm{~nm})$ can be attributed to the formation of block copolymer micelles with no incorporation of OaSPIONs (compare values with those of Table 2). To support this conclusion, aggregates with $1: 1$ weight ratios were prepared for the block copolymer $\mathrm{mPEG}_{45}-b$ $\mathrm{P}_{4 V P_{32}}$. The DLS analysis showed only one particle size distribution, with a maximum intensity peak at $163 \mathrm{~nm}$, showing that increasing the block copolymer to OaSPION ratio can favor the formation of block copolymer micelles without OaSPIONs.

These results show that larger particles were formed when the hydrophobic SPIONs were used. The variation in the size distribution with the composition and with the molecular weight of the block copolymers seems to follow the same trend as that of the hybrid nanoaggregates prepared from hSPIONs by solvent exchange. For the block copolymer having smaller MPEG, there is a maximum particle size in the intermediate $\mathrm{P} 4 \mathrm{VP}$ to $\mathrm{mPEG}$ molar ratio $\left(\mathrm{mPEG}_{45}-b-\mathrm{P} 4 \mathrm{VP}_{56}\right)$. Block copolymers with a greater molecular weight and a greater P4VP to mPEG molar ratio seem to lead to compacted structures, whose particle sizes are similar to those of $\mathrm{mPEG}_{45}-b-\mathrm{P}_{4} \mathrm{VP}_{32}$.

Fig. 4 presents TEM micrographs of SPION aggregates that were prepared from the self-assembly of the $\mathrm{mPEG}_{45}-b-\mathrm{P}_{4} \mathrm{VP}_{32}$ copolymer with OaSPIONs. Compared with the aggregates that were prepared with the hSPIONs, these SPIONs tend to be more densely packed into the core of the aggregates. This effect can be attributed to greater water repulsion by the original coating of these SPIONs.

These results show that with this type of block copolymer it is possible to disperse nanoaggregates of SPIONs in water, if they have either hydrophilic or hydrophobic surfaces. Moreover, the most significant disadvantage in using non-coated iron oxide

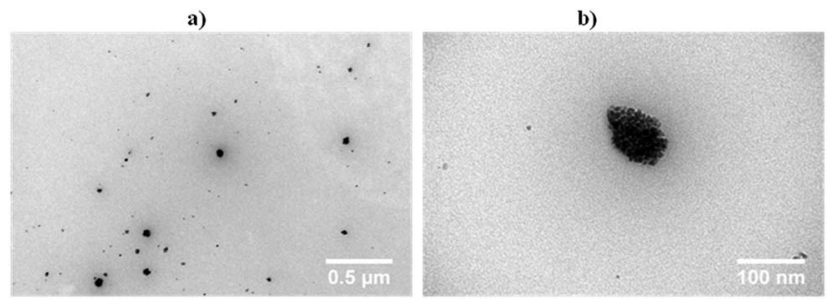

Fig. 4 TEM micrographs of SPION aggregates, prepared with oleic acid-coated SPION nanoaggregates based on the self-assembly of $\mathrm{mPEG}_{45}-b-\mathrm{P}_{4 \mathrm{VP}}$ 32. (a) was taken at a Mag. $\times 60000$ and (b) at Mag. $\times 300000$. nanoparticles is their instability in an aqueous dispersion when kept for long periods of time. This instability arises because of the occurrence of progressive agglomeration. ${ }^{14,18,19}$ In fact, the nanoaggregates prepared by self-assembly with mPEG- $b$-P4VP remained stable for months, independent of the preparation method or the type of SPIONs that were used. However, for further application of these nanoaggregates in specific biological systems, one should evaluate the influence of other medium components in the colloidal stability.

\subsection{Relaxometry of self-assembled MPEG- $b$-P4VP and SPIONs as aqueous dispersions}

SPIONs have been clinically used as $T_{2}$-type (negative) MRI contrast agents, being commercially available under several brand names. ${ }^{48}$ The performance of SPION aggregates, based on their mPEG- $b$-P4VP self-assembly behaviour, as potential MRI contrast agents has been evaluated on the basis of relaxometric measurements. Fig. 5 shows that the paramagnetic contribution to the relaxation rates, $R_{1, \mathrm{p}}$ and $R_{2, \mathrm{p}}$,varied linearly with the Fe concentration and also displays the measured $r_{1}$ and $r_{2}$ relaxivities for a representative dispersion of the hSPION-loaded $\mathrm{mPEG}_{113}-b-\mathrm{P}_{4} \mathrm{VP}_{78}$ and $\mathrm{mPEG}_{45}-b-\mathrm{P}_{4} \mathrm{VP}_{32}$ micelles, prepared respectively by the solvent exchange method and the titration method.

The relaxivity values that were obtained at $20 \mathrm{MHz}$ for different self-assembled systems are summarized in Table 4.

The $r_{1}$ values for MPEG- $b$-P4VP, self-assembled in the presence of SPIONs, are significantly lower than those reported for commercially available standards and for ultrasmall SPIONs (respectively, SSPIONs and USPIONs). ${ }^{28,31,48,49}$ Values of $r_{1}$ are usually interpreted through the use of the outer-sphere Curie relaxation theory, where the accessibility of water molecules to the surface of the nanoparticle is depicted to be a dominant factor, represented by the distance of closest approach. ${ }^{\mathbf{1 2 , 5 0 , 5 1}}$ The hSPION-based micelle dispersions, with constant $10 \mathrm{~nm}$ SPION diameter and hydrodynamic diameters in the 55.8-78.5 $\mathrm{nm}$ range (Table 3), had $r_{1}$ values in the range of 5.1-8.7 $\mathrm{s}^{-1}$ $\mathrm{mM}^{-1}$ Fe. Using citrate-coated single hSPIONs stabilized in water $^{31}$ and other USPIONs in Table 4 as a reference, a
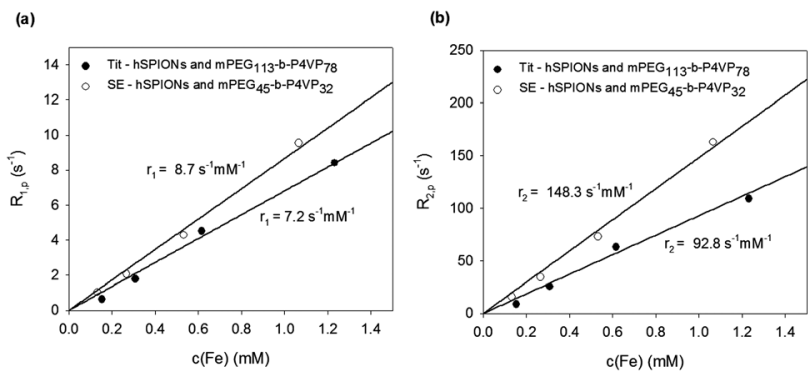

Fig. 5 The paramagnetic contribution to the water proton relaxation rates $\left(20 \mathrm{MHz}, 25^{\circ} \mathrm{C}\right.$ ) (a) for $T_{1}$ and (b) for $T_{2}$ relaxation rates (represented, respectively, as $R_{1, p}$ and $R_{2, p}$ in $\mathrm{s}^{-1}$ ) as a function of iron concentration (in $\mathrm{mM}$ ) for hSPION-loaded-mPEG $45-b$-P4VP32 and $\mathrm{mPEG}_{113}-b-\mathrm{P} 4 \mathrm{VP7} 7 \mathrm{micelles,}$ prepared, respectively, by the titration (Tit) and solvent exchange (SE) methods. 
Table 4 Longitudinal $\left(r_{1}\right)$ and transverse $\left(r_{2}\right)$ relaxivities, and relaxivity ratios $\left(r_{2} / r_{1}\right)$, obtained from relaxometry measurements, for different block copolymers and SPION-self-assembled samples (at $20 \mathrm{MHz}, 25^{\circ} \mathrm{C}, 0.47 \mathrm{~T}$ )

\begin{tabular}{|c|c|c|c|c|c|}
\hline Block copolymer & SPIONs & Method & $r_{1} \pm \sigma\left[\mathrm{s}^{-1} \mathrm{mM}^{-1}\right]$ & $r_{2} \pm \sigma\left[\mathrm{s}^{-1} \mathrm{mM}^{-1}\right]$ & $r_{2} / r_{1}$ \\
\hline & SSPIONs $^{48,49}$ & & $20-40$ & $150-160$ & $4.0-6.0$ \\
\hline & USPIONs $^{28,31,48,49}$ & & $20-30$ & $20-50$ & $1.8-2.1$ \\
\hline $\mathrm{mPEG}_{113}-b-\mathrm{P} 4 \mathrm{VP}_{78}$ & hSPIONs & Tit & $7.2 \pm 0.6$ & $92.8 \pm 7.2$ & 12.8 \\
\hline $\mathrm{mPEG}_{45}-b-\mathrm{P}_{4} \mathrm{VP}_{32}$ & hSPIONs & $\mathrm{SE}$ & $8.7 \pm 0.4$ & $148.3 \pm 7.2$ & 17.1 \\
\hline $\mathrm{mPEG}_{113}-b-\mathrm{P} 4 \mathrm{VP}_{78}$ & hSPIONs & $\mathrm{SE}$ & $5.1 \pm 0.4$ & $96.7 \pm 2.4$ & 19.1 \\
\hline
\end{tabular}

significant reduction in $r_{1}$ was observed for the studied nanoaggregates. They are also smaller than that of ferucarbotran $\left(12.3 \mathrm{~s}^{-1} \mathrm{mM}^{-1} \mathrm{Fe}\right)$ and other SPIONs coated with dextran ${ }^{12}(10-$ $\left.15 \mathrm{~s}^{-1} \mathrm{mM}^{-1} \mathrm{Fe}\right)$ or other sugars ${ }^{52}\left(15-40 \mathrm{~s}^{-1} \mathrm{mM}^{-1} \mathrm{Fe}\right.$ at 20 $\mathrm{MHz}$ ) reported in the literature. The reduction in $r_{1}$ that was observed for the studied nanoaggregates is mainly attributed to a significant decrease in the accessibility of water to the surface of the hSPIONs because of their encapsulation in the hydrophobic mPEG- $b$-P4VP micelle core. For those aggregates that were prepared using the hydrophobic OaSPIONs (of constant 5 $\mathrm{nm}$ size), there was a very significant reduction in $r_{1}$ values, which could be expected due to the increased water hindrance of the original SPIONs. This $r_{1}$ decrease has been observed using other types of polymer-based SPION aggregates..$^{25,27,53-55}$

The hSPION mPEG- $b$-P4VP micelle formulations have $r_{2}$ values in the range of $83.0-148.3 \mathrm{~s}^{-1} \mathrm{mM}^{-1} \mathrm{Fe}$, which are considerably larger than those reported for single hSPIONs stabilized in water, such as citrate-coated single $6.3 \mathrm{~nm}$ hSPIONs $\left(r_{2}=39 \mathrm{~s}^{-1} \mathrm{mM}^{-1} \mathrm{Fe}\right)^{28}$ or CTAB-stabilized SPIONs $\left(23.8 \mathrm{~s}^{-1} \mathrm{mM}^{-1} \mathrm{Fe}\right),{ }^{31}$ or for some USPION formulations, such as some dextran-coated SPIONs (30-50 s$\left.{ }^{-1} \mathrm{mM}^{-1} \mathrm{Fe}\right) .{ }^{49}$ However, they have values that are comparable to or lower than those obtained for other dextran-coated SPIONs $\left(38-189 \mathrm{~s}^{-1} \mathrm{mM}^{-1}\right.$ $\mathrm{Fe}),{ }^{12}$ SPIONS coated with other sugars, ${ }^{52}$ or for systems prepared by self-assembly methodologies..$^{25,27,28,31,53-57}$ They are also much lower than the value $\left(239 \mathrm{~s}^{-1} \mathrm{mM}^{-1} \mathrm{Fe}\right)$ reported for ferucarbotran..$^{12}$ However, a quantitative comparison of published data is difficult due to the different experimental parameters used, such as magnetic field and temperature, which strongly influence the $r_{2}$ values.

Previous studies have established that large $r_{2}$ values are related to a large magnetization of the iron oxide nanocrystals and, thus, with their size. ${ }^{12,58}$ This was experimentally proven for individually encapsulated iron oxide nanocrystals with sizes up to $20 \mathrm{~nm}$ (USPIONs). ${ }^{59}$ As the maximum relaxivity for USPIONs is only expected by theory at a particle size of $25-27 \mathrm{~nm},{ }^{60}$ which so far cannot be synthesized, higher $r_{2}$ values can only be achieved through clustering of USPIONs to larger clusters. Large $r_{2}$ increases have been achieved for commercially available products (SSPIONs, such as Resovist or Endorem, Table 4), ${ }^{48,49}$ and for SPION aggregates encapsulated in liposomes, ${ }^{61}$ or within polymeric micellar cores, due to the collective magnetic effects of the nanoparticle assemblies. This effect is enhanced by the increased SPIONs' diameter and an increased loading density and varies in a complex way with the size of the nanocluster, ${ }^{58}$ as shown by recent theoretical descriptions. ${ }^{12,50,60,62-64}$ The observed $r_{2}$ values may have a complex dependence on the extent of SPION encapsulation in the micelle core and on their water accessibility, which is determined by the adopted selfassembly methodology. The increased dispersibility of the aggregates prepared by the solvent exchange method relative to the titration method significantly increased $r_{2}$ when the block copolymers containing shorter $\mathrm{mPEG}$ blocks were used, but had no significant effects for the micelles with the longer MPEG blocks.

The nanoaggregates that were prepared with OaSPIONs gave $r_{2}$ values in the range of $58.0-97.5 \mathrm{~s}^{-1} \mathrm{mM}^{-1} \mathrm{Fe}$. These results suggest that, in spite of the extensive SPION aggregation observed for these systems, the greater hindrance of the iron oxide particles in OaSPION-based dispersions, resulting from their stronger hydrophobic character, may decrease $r_{2}$.

The relaxivity ratio, $r_{2} / r_{1}$, is another important parameter to evaluate the efficiency of $T_{2}$-type contrast agents, which increases with the value of this ratio by limiting $T_{1}$-weighting effects. The $r_{2} / r_{1}$ ratio of the micelle preparations studied is relatively high, making these systems potentially very efficient $T_{2}$ MRI contrast agents. These studies confirm that SPION clustering, arising from their incorporation into the hydrophobic mPEG- $b$-P4VP micelle's core, enhances $r_{2}$ and $r_{2} / r_{1}$ relaxivity ratios, but this effect may be influenced by the
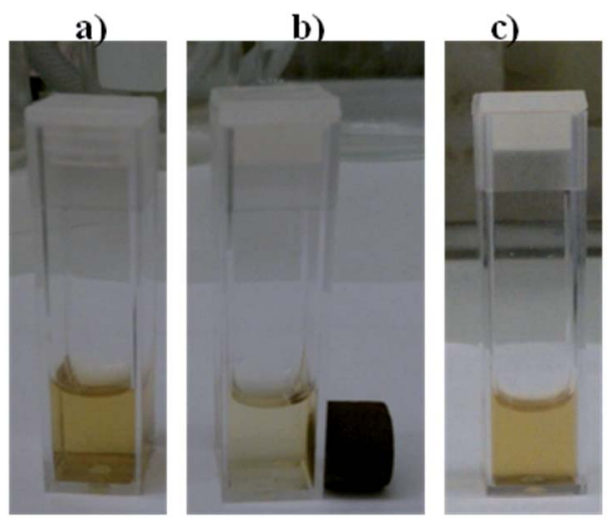

Fig. 6 Comparison of aqueous dispersions of hSPION-loaded $\mathrm{mPEG}_{45}-b-\mathrm{P} \mathrm{VP}_{32}$ micelles (a) before, (b) during, and (c) after being subjected to an external magnetic field. 
hindered water accessibility to the micelle core and the degree of clustering. ${ }^{25,27,28,53-55}$

Moreover, the SPIONs' nanoaggregates were shown to remain stable in the aqueous dispersions after being subjected to a magnetic field. Fig. 6 demonstrates the susceptibility of the hSPION-loaded mPEG $_{45}-b$-P4VP32 micelles to the influence of an applied external magnetic field. When the magnetic field was removed, the particles became well dispersed in water again. Also, there was no evidence of differences in the quality of the dispersion, when compared to the dispersions that had not been subjected to the external magnetic field.

\section{Conclusions}

It has been shown that mPEG- $b$-P4VP block copolymers can be used to prepare hybrid nanoaggregates of SPIONs, independent of their original hydrophilic or hydrophobic coating, by selfassembly in aqueous media. Well-defined block copolymers having different compositions and molecular weights were prepared by ATRP methodologies and were successfully used to prepare micelle structures in aqueous media. The type of structures that were formed was dependent on the preparation method, giving rise to much larger structures when prepared using a titration method (from 24.7 to $613 \mathrm{~nm}$ ) than by a solvent exchange method (from 17.6 to $35.7 \mathrm{~nm}$ ). Similar behavior was observed when the self-assembly was carried out in the presence of SPIONs. The block copolymer was capable of encapsulating the SPIONs with hydrophilic or hydrophobic nature in the micelle's core. The obtained hybrid SPION nanoaggregates perform as efficient $T_{2}$-weighted magnetic resonance imaging contrast agents, their $r_{1}$ values being significantly lower and $r_{2}$ in a similar range to those of commercially available SPIONs, giving rise to SPION aggregates that possess large relaxivity ratios $\left(r_{2} / r_{1}\right)$. The aggregates prepared in this study maintained a good stability in water for long periods of time, contrary to what occurs with native SPIONs, and after being subjected to an external magnetic field.

\section{Acknowledgements}

This work was financially supported by Fundação para a Ciência e a Tecnologia (Portugal), project Nanohybrid (PTDC/EQU-EPR/ 114354/2009) and project PEst-C/SAU/LA0001/2011, by Rede Nacional de RMN (REDE/1517/RMN/2005) with the acquisition of the Bruker Avance III $400 \mathrm{MHz}$ NMR spectrometer in Coimbra (FEDER) and by the EU COST TD1004 Action "Theragnostics Imaging and Therapy”. Nuno Rocha acknowledges FCT-MCTES for his postdoctoral scholarship (SFRH/BPD/86352/2012).

\section{References}

1 Z. H. Nie, A. Petukhova and E. Kumacheva, Properties and emerging applications of self-assembled structures made from inorganic nanoparticles, Nat. Nanotechnol., 2010, 5(1), 15-25.
2 P. Alexandridis and M. Tsianou, Block copolymer-directed metal nanoparticle morphogenesis and organization, Eur. Polym. J., 2011, 47(4), 569-583.

3 W. A. Braunecker and K. Matyjaszewski, Controlled/living radical polymerization: Features, developments, and perspectives, Prog. Polym. Sci., 2007, 32(1), 93-146.

4 K. Matyjaszewski, Atom Transfer Radical Polymerization: From Mechanisms to Applications, Isr. J. Chem., 2012, 52(3-4), 206-220.

5 K. Matyjaszewski and N. V. Tsarevsky, Nanostructured functional materials prepared by atom transfer radical polymerization, Nat. Chem., 2009, 1(4), 276-288.

6 R. A. Cordeiro, N. Rocha, J. P. Mendes, et al., Synthesis of well-defined poly(2-(dimethylamino)ethyl methacrylate) under mild conditions and its co-polymers with cholesterol and PEG using $\mathrm{Fe}(0) / \mathrm{Cu}(\mathrm{II})$ based SARA ATRP, Polym. Chem., 2013, 4(10), 3088-3097.

7 N. Rocha, P. V. Mendonca, J. P. Mendes, et al., Facile Synthesis of Well-Defined Telechelic Alkyne-Terminated Polystyrene in Polar Media Using ATRP With Mixed $\mathrm{Fe} / \mathrm{Cu}$ Transition Metal Catalyst, Macromol. Chem. Phys., 2013, 214(1), 76-84.

8 N. Rocha, J. F. J. Coelho, B. Barros, et al., Deviation from the theoretical predictions in the synthesis of amphiphilic block copolymers in a wide range of compositions based on poly(vinyl chloride) by single electron transfer: Degenerative chain living radical polymerization in suspension medium, J. Appl. Polym. Sci., 2013, 127(5), 3407-3417.

9 C. M. R. Abreu, P. V. Mendonca, A. C. Serra, et al., Inorganic Sulfites: Efficient Reducing Agents and Supplemental Activators for Atom Transfer Radical Polymerization, ACS Macro Lett., 2012, 1(11), 1308-1311.

10 Y. B. Liu and X. S. Wang, Recent advances in block copolymer-assisted synthesis of supramolecular inorganic/ organic hybrid colloids, Polym. Chem., 2011, 2(12), 27412757.

11 A.-H. Lu, E. L. Salabas and F. Schueth, Magnetic nanoparticles: Synthesis, protection, functionalization, and application, Angew. Chem., Int. Ed., 2007, 46(8), 1222-1244.

12 S. Laurent, D. Forge, M. Port, et al., Magnetic iron oxide nanoparticles: Synthesis, stabilization, vectorization, physicochemical characterizations, and biological applications, Chem. Rev., 2008, 108(6), 2064-2110.

13 A. K. Gupta and M. Gupta, Synthesis and surface engineering of iron oxide nanoparticles for biomedical applications, Biomaterials, 2005, 26(18), 3995-4021.

14 M. Mahmoudi, S. Sant, B. Wang, S. Laurent and T. Sen, Superparamagnetic iron oxide nanoparticles (SPIONs): Development, surface modification and applications in chemotherapy, Adv. Drug Delivery Rev., 2011, 63(1-2), 24-46.

15 N. Wang, Y. Guan, L. Yang, et al., Magnetic nanoparticles (MNPs) covalently coated by PEO-PPO-PEO block copolymer for drug delivery, J. Colloid Interface Sci., 2013, 395, 50-57.

16 M. F. Casula, A. Corrias, P. Arosio, et al., Design of waterbased ferrofluids as contrast agents for magnetic 
resonance imaging, J. Colloid Interface Sci., 2011, 357(1), 5055.

17 S.-B. Seo, J. Yang, T.-I. Lee, et al., Enhancement of magnetic resonance contrast effect using ionic magnetic clusters, $J$. Colloid Interface Sci., 2008, 319(2), 429-434.

$18 \mathrm{~N}$. Lee and T. Hyeon, Designed synthesis of uniformly sized iron oxide nanoparticles for efficient magnetic resonance imaging contrast agents, Chem. Soc. Rev., 2012, 41(7), 2575-2589.

19 J. K. Oh and J. M. Park, Iron oxide-based superparamagnetic polymeric nanomaterials: Design, preparation, and biomedical application, Prog. Polym. Sci., 2011, 36(1), 168189.

20 L. E. Euliss, S. G. Grancharov, S. O'Brien, et al., Cooperative assembly of magnetic nanoparticles and block copolypeptides in aqueous media, Nano Lett., 2003, 3(11), 1489-1493.

21 B. S. Kim, J. M. Qiu, J. P. Wang and T. A. Taton, Magnetomicelles: Composite nanostructures from magnetic nanoparticles and cross-linked amphiphilic block copolymers, Nano Lett., 2005, 5(10), 1987-1991.

22 J. F. Berret, A. Sehgal, M. Morvan, O. Sandre, A. Vacher and M. Airiau, Stable oxide nanoparticle clusters obtained by complexation, J. Colloid Interface Sci., 2006, 303(1), 315-318.

23 S. B. Lecommandoux, O. Sandre, F. Checot, J. RodriguezHernandez and R. Perzynski, Magnetic nanocomposite micelles and vesicles, Adv. Mater., 2005, 17(6), 712-718.

24 M. Lattuada and T. A. Hatton, Preparation and controlled self-assembly of janus magnetic nanoparticles, J. Am. Chem. Soc., 2007, 129(42), 12878-12889.

25 H. Ai, C. Flask, B. Weinberg, et al., Magnetite-loaded polymeric micelles as ultrasensitive magnetic-resonance probes, Adv. Mater., 2005, 17(16), 1949-1956.

26 G.-B. Hong, J.-X. Zhou and R.-X. Yuan, Folate-targeted polymeric micelles loaded with ultrasmall superparamagnetic iron oxide: combined small size and high MRI sensitivity, Int. J. Nanomed., 2012, 7, 2863-2872.

27 D. Chen, X. Xia, H. Gu, et al., pH-responsive polymeric carrier encapsulated magnetic nanoparticles for cancer targeted imaging and delivery, J. Mater. Chem., 2011, 21(34), 12682-12690.

28 J. F. Berret, N. Schonbeck, F. Gazeau, et al., Controlled clustering of superparamagnetic nanoparticles using block copolymers: Design of new contrast agents for magnetic resonance imaging, J. Am. Chem. Soc., 2006, 128(5), 17551761.

29 R. Sondjaja, T. A. Hatton and M. K. C. Tam, Clustering of magnetic nanoparticles using a double hydrophilic block copolymer, poly(ethylene oxide)-b-poly(acrylic acid), $J$. Magn. Magn. Mater., 2009, 321(16), 2393-2397.

30 S. R. Wan, Y. Zheng, Y. Q. Liu, H. S. Yan and K. L. Liu, $\mathrm{Fe}_{3} \mathrm{O}_{4}$ nanoparticles coated with homopolymers of glycerol mono(meth) acrylate and their block copolymers, J. Mater. Chem., 2005, 15(33), 3424-3430.

31 J. Hu, Y. Qian, X. Wang, T. Liu and S. Liu, Drug-Loaded and Superparamagnetic Iron Oxide Nanoparticle SurfaceEmbedded Amphiphilic Block Copolymer Micelles for
Integrated Chemotherapeutic Drug Delivery and MR Imaging, Langmuir, 2012, 28(4), 2073-2082.

32 M. Aizawa and J. M. Buriak, Block copolymer templated chemistry for the formation of metallic nanoparticle arrays on semiconductor surfaces, Chem. Mater., 2007, 19(21), 5090-5101.

33 N. G. Kang, B. G. Kang, H. D. Koh, M. Changez and J. S. Lee, Block copolymers containing pyridine moieties: Precise synthesis and applications, React. Funct. Polym., 2009, 69(7), 470-479.

34 A. Fahmi, T. Pietsch, C. Mendoza and N. Cheval, Functional hybrid materials, Mater. Today, 2009, 12(5), 44-50.

35 J. H. Koh, J. A. Seo, J. T. Park and J. H. Kim, Synthesis and characterization of $\mathrm{AgBr}$ nanocomposites by templated amphiphilic comb polymer, J. Colloid Interface Sci., 2009, 338(2), 486-490.

36 W. Q. Zhang, L. Q. Shi, R. J. Ma, Y. L. An, Y. L. Xu and K. Wu, Micellization of thermo- and pH-responsive triblock copolymer of poly(ethylene glycol)- $b$-poly(4-vinylpyridine)- $b$ poly( $N$-isopropylacrylamide), Macromolecules, 2005, 38(21), 8850-8852.

37 S. Mendrek, A. Mendrek, H.-J. Adler, A. Dworak and D. Kuckling, Synthesis and Characterization of $\mathrm{pH}$ Sensitive Poly(glycidol)- $b$-poly(4-vinylpyridine) Block Copolymers, J. Polym. Sci., Part A: Polym. Chem., 2009, 47(7), 1782-1794.

38 H. Liu, R. Shi, W. Wan, R. Yang and Y. Wang, A well-defined diblock copolymer of poly(ethylene oxide)-block-poly(4vinylpyridine) for separation of basic proteins by capillary zone electrophoresis, Electrophoresis, 2008, 29(13), 28122819.

39 L. Zhao, R. Ma, J. Li, Y. Li, Y. An and L. Shi, J- and HAggregates of 5,10,15,20-Tetrakis-(4-sulfonatophenyl)porphyrin and Interconversion in PEG- $b$-P4VP Micelles, Biomacromolecules, 2008, 9(10), 2601-2608.

40 T. Azzam, L. Bronstein and A. Eisenberg, Water-soluble surface-anchored gold and palladium nanoparticles stabilized by exchange of low molecular weight ligands with biamphiphilic triblock copolymers, Langmuir, 2008, 24(13), 6521-6529.

41 J. Thevenot, H. Oliveira, O. Sandre and S. Lecommandoux, Magnetic responsive polymer composite materials, Chem. Soc. Rev., 2013, 42(17), 7099-7116.

42 M. Ciampolini and N. Nardi, Five-Coordinated High-Spin Complexes of Bivalent Cobalt, Nickel, and Copper with Tris(2-dimethylaminoethyl)amine, Inorg. Chem., 1966, 5(1), 41-44.

43 K. Jankova, X. Y. Chen, J. Kops and W. Batsberg, Synthesis of amphiphilic PS- $b$-PEG- $b$-PS by atom transfer radical polymerization, Macromolecules, 1998, 31(2), 538-541.

44 K. R. M. Vidts and F. E. Du Prez, Design of water-soluble block copolymers containing poly(4-vinylpyridine) by atom transfer radical polymerization, Eur. Polym. J., 2006, 42(1), 43-50.

45 H. Maleki, A. Simchi, M. Imani and B. F. O. Costa, Sizecontrolled synthesis of superparamagnetic iron oxide nanoparticles and their surface coating by gold for 
biomedical applications, J. Magn. Magn. Mater., 2012, 324(23), 3997-4005.

46 N. A. Lynd, A. J. Meuler and M. A. Hillmyer, Polydispersity and block copolymer self-assembly, Prog. Polym. Sci., 2008, 33(9), 875-893.

47 T. Mauser, C. Dejugnat and G. B. Sukhorukov, Balance of hydrophobic and electrostatic forces in the $\mathrm{pH}$ response of weak polyelectrolyte capsules, J. Phys. Chem. B, 2006, 110(41), 20246-20253.

48 C. F. G. C. Geraldes and S. Laurent, Classification and basic properties of contrast agents for magnetic resonance imaging, Contrast Media Mol. Imaging, 2009, 4(1), 1-23.

49 Y. X. J. Wang, S. M. Hussain and G. P. Krestin, Superparamagnetic iron oxide contrast agents: physicochemical characteristics and applications in MR imaging, Eur. J. Radiol., 2001, 11(11), 2319-2331.

50 A. Roch, R. N. Muller and P. Gillis, Theory of proton relaxation induced by superparamagnetic particles, J. Chem. Phys., 1999, 110(11), 5403-5411.

51 S. L. C. Pinho, S. Laurent, J. Rocha, et al., Relaxometric Studies of $\gamma-\mathrm{Fe}_{2} \mathrm{O}_{3} @ \mathrm{SiO}_{2}$ Core Shell Nanoparticles: When the Coating Matters, J. Phys. Chem. C, 2011, 116(3), 22852291.

52 L. Lartigue, C. Innocenti, T. Kalaivani, et al., WaterDispersible Sugar-Coated Iron Oxide Nanoparticles. An Evaluation of their Relaxometric and Magnetic Hyperthermia Properties, J. Am. Chem. Soc., 2011, 133(27), 10459-10472.

53 E. S. Guang Choo, X. Tang, Y. Sheng, B. Shuter and J. Xue, Controlled loading of superparamagnetic nanoparticles in fluorescent nanogels as effective T2-weighted MRI contrast agents, J. Mater. Chem., 2011, 21(7), 2310-2319.

54 J.-R. Lai, Y.-W. Chang, H.-C. Yen, et al., Multifunctional doxorubicin/superparamagnetic iron oxide-encapsulated Pluronic F127 micelles used for chemotherapy/magnetic resonance imaging, J. Appl. Phys., 2010, 107(9), 09B318.
55 H. Tan, J. M. Xue, B. Shuter, X. Li and J. Wang, Synthesis of PEOlated $\mathrm{Fe}_{3} \mathrm{O}_{4} @ \mathrm{SiO}_{2}$ Nanoparticles via Bioinspired Silification for Magnetic Resonance Imaging, Adv. Funct. Mater., 2010, 20(5), 722-731.

56 J. Kim, J. E. Lee, S. H. Lee, et al., Designed Fabrication of a Multifunctional Polymer Nanomedical Platform for Simultaneous Cancer-Targeted Imaging and Magnetically Guided Drug Delivery, Adv. Mater., 2008, 20(3), 478-483.

57 D. Niu, Y. Li, Z. Ma, et al., Preparation of Uniform, WaterSoluble, and Multifunctional Nanocomposites with Tunable Sizes, Adv. Funct. Mater., 2010, 20(5), 773-780.

58 E. Pöselt, H. Kloust, U. Tromsdorf, et al., Relaxivity Optimization of a PEGylated Iron-Oxide-Based Negative Magnetic Resonance Contrast Agent for T2-Weighted SpinEcho Imaging, ACS Nano, 2012, 6(2), 1619-1624.

59 S. Mornet, S. Vasseur, F. Grasset and E. Duguet, Magnetic nanoparticle design for medical diagnosis and therapy, $J$. Mater. Chem., 2004, 14(14), 2161-2175.

60 P. Gillis, F. Moiny and R. A. Brooks, On T2-shortening by strongly magnetized spheres: A partial refocusing model, Magn. Reson. Med., 2002, 47(2), 257-263.

61 A. A. Bogdanov Jr, C. Martin, R. Weissleder and T. J. Brady, Trapping of dextran-coated colloids in liposomes by transient binding to aminophospholipid: preparation of ferrosomes, Biochim. Biophys. Acta, Biomembr., 1994, 1193(1), 212-218.

62 R. A. Brooks, T2-shortening by strongly magnetized spheres: A chemical exchange model, Magn. Reson. Med., 2002, 47(2), 388-391.

63 A. Roch, Y. Gossuin, R. N. Muller and P. Gillis, Superparamagnetic colloid suspensions: Water magnetic relaxation and clustering, J. Magn. Magn. Mater., 2005, 293(1), 532-539.

64 Q. L. Vuong, P. Gillis and Y. Gossuin, Monte Carlo simulation and theory of proton NMR transverse relaxation induced by aggregation of magnetic particles used as MRI contrast agents, J. Magn. Reson., 2011, 212(1), 139-148. 